American J. of Engineering and Applied Sciences 3 (1): 207-213, 2010

ISSN 1941-7020

(C) 2010 Science Publications

\title{
Modification Approach of Fuzzy Logic Model for Predicting of Cutting Force When Machining Nickel Based Hastelloy C-276
}

\author{
${ }^{1}$ Basim A. Khidhir, ${ }^{1}$ Bashir Mohamed and ${ }^{2}$ Mahmoud A. A. Younis \\ ${ }^{1}$ Department of Mechanical Engineering, University Tenaga National, \\ Km 7, Jalan Kajang-Puchong, 43009 Kajang, Selangor, Malaysia \\ ${ }^{2}$ Program of Electrical Engineering, University Industri Selangor, \\ Bestari Jaya, 45000, Kuala Selangor, Selangor, Malaysia
}

\begin{abstract}
Problem statement: Most Nickel based Hastelloy C-276 is a difficult-to-machine material because of its low thermal diffusive property and high strength at high temperature. Machinability consideration of nickel based Hastelloy C-276 in turning operations has been carried out using ceramic inserts under dry conditions. Approach: This study described a modification approach applied to a fuzzy logic based model for predicting cutting force where the machining parameters for cutting speed ranges, feed rate, depth of cut and approach angle are not overlapping. For this study, data were selected depending on the design of experiments. Response surface methodology was applied to predict the cutting force and to examine the fuzzy logic based model. Results: The modification approach fuzzy logic based model produced the cutting force data providing good correlation with response surface data. In this situation the cutting force data were superimposed and results were adjusted according to their own ranges. Conclusion: A review of literatures on optimization techniques revealed that there were, in particular, successful industrial applications of design of experiment-based approaches for optimal settings of process variables.
\end{abstract}

Key words: Cutting speed, surface response methodology, first order (linear + interaction), fuzzy logic

\section{INTRODUCTION}

Manufacturing industries have long depended on the skill and experience of the operators for optimal selection of cutting conditions and cutting tools. Considerable efforts are still in progress on the use of handbook based conservative cutting conditions and cutting tool selection at the process planning level. The most adverse effect of such a not-very scientific practice is decreased productivity due to sub-optimal use of machining capability. The need for selecting and implementing optimal machining conditions and the most suitable cutting tool has been felt over the last few decades. Progress has been slow since all the process parameters need to be optimized. Furthermore, for realistic solutions, the many constraints met in practice, such as low machine tool power, torque, force limits and component surface roughness must be overcome. The non-availability of the required technological performance equation represents a major obstacle to implementation of optimized cutting conditions in practice. This follows since extensive testing is required to establish empirical performance equations for each tool cutting-work material combination for a given machining operation, which can be quite expensive when a wide spectrum of machining operations is considered. However, in this study to get the sufficient model that related the cutting force and the cutting parameters (cutting speed, feed rate, depth of cut and approach angle), different tests for each and every combination of cutting tools and workpiece material needed. Several of cutting speeds, feed rates, depth of cuts and approach angles have been taken into account to get the module that predict the cutting force by using response surface methodology. Experimental results were used for modeling using Response Surface Methodology (RSM) (Montgomery, 2001). The RSM is practical, economical and relatively easy for use and it was used by many researchers (Mead and Pike, 1975; Hill and Hunter, 1966) reviewed the earliest work on response surface methodology (El Baradie, 1993; Sundaram and Lambert, 1981; Hasegawa et al., 1976) for modeling machining processes. Response Surface Methodology (RSM) is a combination of experimental

Corresponding Author: Basim A. Khidhir, Department of Mechanical Engineering, University Tenaga National, Km 7, Jalan Kajang-Puchong, 43009 Kajang, Selangor, Malaysia 
and regression analysis and statistical inferences. The concept of a response surface involves a dependent variable $y$ called the response variable and several independent variables $\mathrm{x}_{1}, \mathrm{x}_{2}, \ldots, \mathrm{x}_{\mathrm{k}}$ If all of these variables are assumed to be measurable, the response surface can be expressed as:

$\mathrm{y}=\mathrm{f}\left(\mathrm{x}_{1} ; \mathrm{x}_{2} ; \ldots ; \mathrm{x}_{\mathrm{k}}\right)$

Optimizing the response variable $y$, it is assumed that the independent variables are continuous and controllable by the experimenter with negligible error. The response or the dependent variable is assumed to be a random variable. In our experiments turning operation was selected because it's the most basic cutting process. It is necessary to find a suitable combination of cutting speed $\left(x_{1}=\ln V\right)$, feed rate $\left(x_{2}=\ln f\right)$, depth of cut $\left(x_{3}=\ln \right.$ d) and approach angle $\left(x_{4}=\ln K\right)$ that optimize cutting force $(\mathrm{y}=\ln \mathrm{F})$. The observed response $\mathrm{y}$ as a function of the speed, feed, depth of cut and approach angle can be written as:

$\mathrm{y}=\mathrm{f}\left(\mathrm{x}_{1} ; \mathrm{x}_{2} ; \mathrm{x}_{3}\right)+\varepsilon$

Usually a low order polynomial (first-order and second-order) in some regions of the independent variables is employed. The linear + interactions model:

$$
\begin{gathered}
y=\beta_{0}+\sum_{n=1}^{\infty}\left(\beta_{i} x_{i}\right)+\sum_{j}\left(\beta_{i j} x_{i} x j\right)+\varepsilon \\
\text { for } i<j
\end{gathered}
$$

are generally utilized in RSM problems. The parameters $\beta$ of the polynomials are estimated by the method of least squares. The proposed relationship between the machining responses (cutting force) and machining independent variables can be represented by the following:

$$
\begin{aligned}
y= & \beta_{0} x_{0}+\beta_{1} x_{1}+\beta_{2} x_{2}+\beta_{3} x_{3}+\beta_{4} x_{4}+\beta_{5} x_{1} x_{2} \\
& +\beta_{6} x_{1} x_{3}+\beta_{7} x_{1} x_{4}+\beta_{8} x_{2} x_{3}+\beta_{9} x_{2} x_{4}+\beta_{10} x_{3} x_{4}
\end{aligned}
$$

where, $y$ is the cutting force:

$\mathrm{x}_{\mathrm{o}}=1$ (dummy variables)

$\mathrm{x}_{1}=\ln \mathrm{V}$

$\mathrm{x}_{2}=\ln \mathrm{f}$

$\mathrm{x}_{3}=\ln (\mathrm{doc})$

$\mathrm{x}_{4}=\ln \mathrm{K}$

$\varepsilon=\ln \varepsilon$

where, $\varepsilon$ is assumed to be normally-distributed uncorrelated random error with zero mean and constant variance, $\beta_{\mathrm{o}}=\ln \mathrm{C}$ and $\beta_{1}, \beta_{2}, \beta_{3}$ and $\beta_{10}$ are the model parameters.

\section{MATERIALS AND METHODS}

Experimental set-up: A Kistler force three component dynamometer (Type 9215A1, calibrated range: Fx. $0 \pm 5000 \mathrm{~N}$, Fy. $0 \pm 5000 \mathrm{~N}$ and Fz. $0 \pm 3000 \mathrm{~N}$ ) in conjunction with three Kistler charge amplifiers (Type 5070), used to convert the dynamometer output signal into a voltage signal appropriate for the data acquisition system and a computer were used to measure and record the cutting forces. OKUMA CNC turning machine supported with Spindle Drive motor $11 \mathrm{KW}$ and $6000 \mathrm{Rpm}$ maximum speed. Z-axis Simens AC Servo motor $8 \mathrm{Nm}$ and $\mathrm{X}$ - axis Simens motor $6 \mathrm{Nm}$ as shown in Fig. 1. High speed camera type sports Cam 250 and sports Cam 250, resolution 640×480, recording rate 250 , total frame 4,368 and recording time $17 \mathrm{sec}$. as shown in Fig. 2.

Work material: The work material used as the test specimen was Hastelloy C-276. Two cylindrical bars of Hastelloy (500 $\mathrm{mm}$ long and $57.15 \mathrm{~mm}$ diameter) were used for the tests. Details of the material properties are given in Table 1 and 2. The nickel based alloy round bar to ASTM B574-99a specification was purchased from Hynes international, INC. The material annealed at $1120^{\circ} \mathrm{C}$ (held $75 \mathrm{~min}$ ) and water quenched.

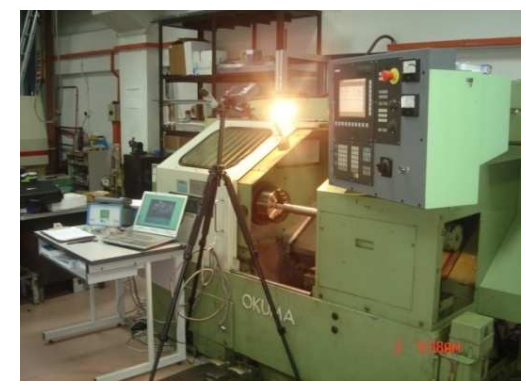

Fig. 1: CNC machine used in experiments

Table 1: Chemical composition

\begin{tabular}{lc}
\hline Components & Percentage \\
\hline $\mathrm{Ni}$ & 57.000 \\
$\mathrm{Co}$ & 1.620 \\
$\mathrm{Cr}$ & 15.440 \\
$\mathrm{Mo}$ & 15.340 \\
$\mathrm{Fe}$ & 5.430 \\
$\mathrm{~W}$ & 3.670 \\
$\mathrm{~V}$ & 0.410 \\
$\mathrm{Mn}$ & 0.520 \\
$\mathrm{C}$ & 0.004 \\
Others & $\mathrm{Si}<0.02 ; \mathrm{P}-0.005 ; \mathrm{S}<0.01$ \\
\hline
\end{tabular}

Table 2: Physical properties

\begin{tabular}{ll}
\hline Density & $8.89 \mathrm{~g} \mathrm{~cm}^{-3}$ \\
Electrical Resistively & $1.3 \mu \Omega-\mathrm{m}$ \\
Dynamic modulus of elasticity & $229 \mathrm{MPa}$ \\
Thermal conductivity & $10.2 \mathrm{~W} \mathrm{~m}^{-1} \mathrm{~K}$ \\
Specific heat & $427 \mathrm{~J} \mathrm{Kg}^{-1} \mathrm{~K}$ \\
\hline
\end{tabular}


Am. J. Engg. \& Applied Sci., 3 (1): 207-213, 2010

Table 3: Insert and tool holder information

\begin{tabular}{llllllr} 
Sq. & Insert code & Tool holder & Approach angle & Rake angle & $\begin{array}{l}\text { Inclination } \\
\text { angle } \lambda(\mathrm{deg})\end{array}$ & $\begin{array}{l}\text { Nose } \\
\text { radius (mm) }\end{array}$ \\
\hline 1 & RNGN 120400E & CRSNR 2525M 12-ID & 0 & -6 & -6 & 12.0 \\
2 & SNGN 120412E & SNGN 120412E & 45 & -6 & -6 & 1.2 \\
3 & CNGN 120408 E & CCLNR 2525M 12-4 & 95 & -6 & -6 & 0.8 \\
\hline
\end{tabular}

Table 4: Variables coding identifications

\begin{tabular}{llll}
\hline Code & -1 & 0 & +1 \\
\hline${\text { Cutting speed, } \mathrm{m} \mathrm{min}^{-1}}_{\text {Feed rate, } \mathrm{mm} \mathrm{rev}^{-1}}$ & 150 & 200 & 250 \\
Depth of cut, mm & 0.15 & 0.2 & 0.25 \\
Approach angle, deg. & 0.5 & 1 & 1.5 \\
\hline
\end{tabular}

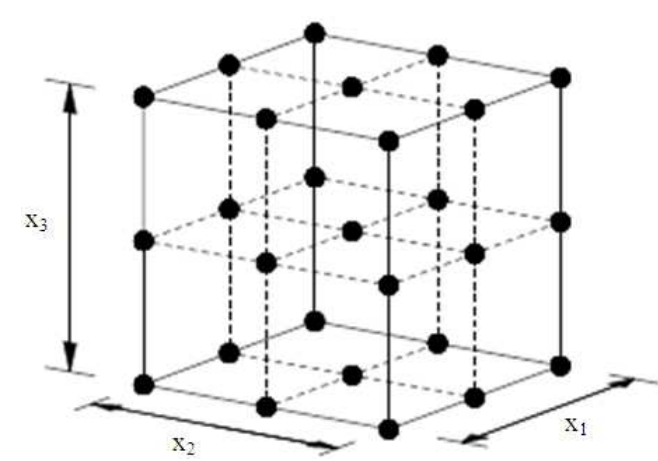

Fig. 2: The $3 \mathrm{~N}$ full factorial

Tool material: Ceramic inserts were used for the turning tests. These inserts are manufactured by Sandvik. The Insert code and Tool holder information listed on Table 3.

Design of experiment: A commercial statistical analysis software "Minitab" was employed for design of experiment. In Minitab, RSM is used to find a combination of factors which gives the optimal response. RSM is actually a collection of mathematical and statistical technique that is useful for the modeling and analysis of problems in which a response of interest is influenced by several variables and the objectives is to optimize the response (Montgomery, 2001). There are essentially two main types of designs experiments which are based on response surface analysis as follows:

\section{- $\quad$ Central Composite Design (CCD) \\ - Box-Behnken Design (BBD)}

Both of these methodologies require a quadratic relationship between the experimental factor and the responses. In this study the BBD has been chosen as shown in Fig. 2.

The levels of independent variables and coding identifications used in this design are presented in Table 4.
Table 5: Experimental conditions and results obtained using ceramic inserts

\begin{tabular}{lccccc}
\hline $\begin{array}{l}\text { Run } \\
\text { order }\end{array}$ & $\begin{array}{l}\text { Cutting } \\
\text { speed, } \\
\left(\mathrm{m} \mathrm{min}^{-1}\right)\end{array}$ & $\begin{array}{l}\text { Feed } \\
\text { rate, } \\
\left(\mathrm{mm} \mathrm{rev}^{-1}\right)\end{array}$ & $\begin{array}{l}\text { Depth } \\
\text { of cut, } \\
\mathrm{d}(\mathrm{mm})\end{array}$ & $\begin{array}{l}\text { Approach } \\
\text { angle, } \\
\mathrm{K}(\mathrm{deg})\end{array}$ & $\begin{array}{l}\text { Exp. } \\
\text { Cutting } \\
\text { force, } \mathrm{F}(\mathrm{N})\end{array}$ \\
\hline 1 & 0 & -1 & 0 & -1 & 963.20 \\
2 & 1 & 1 & 0 & 0 & 1627.80 \\
3 & 0 & 0 & -1 & -1 & 695.27 \\
4 & 0 & -1 & -1 & 0 & 467.60 \\
5 & 1 & 0 & 0 & 1 & 1731.90 \\
6 & 1 & 0 & -1 & 0 & 640.80 \\
7 & 0 & 0 & -1 & 1 & 1017.00 \\
8 & 0 & 1 & 0 & -1 & 1452.00 \\
9 & -1 & 0 & 1 & 0 & 3053.70 \\
10 & -1 & 0 & 0 & 1 & 2077.00 \\
11 & 0 & -1 & 0 & 1 & 1592.60 \\
12 & 0 & 1 & -1 & 0 & 781.10 \\
13 & 1 & 0 & 1 & 0 & 2402.60 \\
14 & 0 & 1 & 1 & 0 & 2652.50 \\
15 & 0 & 1 & 0 & 1 & 2449.60 \\
16 & 1 & -1 & 0 & 0 & 766.50 \\
17 & 1 & 0 & 0 & -1 & 1536.30 \\
18 & -1 & -1 & 0 & 0 & 922.00 \\
19 & 0 & -1 & 1 & 0 & 2240.00 \\
20 & 0 & 0 & 0 & 0 & 1844.00 \\
21 & 0 & 0 & 1 & -1 & 2161.50 \\
22 & 0 & 0 & 1 & 1 & 2805.80 \\
23 & -1 & 0 & -1 & 0 & 1041.20 \\
24 & 0 & 0 & 0 & 0 & 1835.20 \\
25 & -1 & 0 & 0 & -1 & 1569.70 \\
26 & 0 & 0 & 0 & 0 & 1844.50 \\
27 & -1 & 1 & 0 & 0 & 1896.00 \\
\hline & & 0 & 0 & &
\end{tabular}

Table 5 shows the experimental conditions and results obtained using ceramic inserts. All of the turning tests were run dry. Box-Behnken Design is normally used when performing non-sequential experiments. That is, performing the experiment only once. These designs allow efficient estimation of the first-order coefficients. Because Box-Behnken Design has fewer design points, they are less expensive to run than central composite designs with the same number of factors. Box-Behnken Design do not have axial points, thus can be sure that all design points fall within the safe operating. Box-Behnken Design also ensures that all factors are never set at their high levels simultaneously (Box and Wilson, 1951; Box and Hunter, 1957; Box and Youle, 1955). Figure 3 shows the $3 \mathrm{~N}$ full factors Box-Behnken. Preliminary tests were carried out to find the suitable cutting speed V, federate $\mathrm{f}$, depth of cut $\mathrm{d}$ and approach angle $\mathrm{K}$ as shown in Table 4. Every one passes (one pass is equal to $20 \mathrm{~mm}$ ), the cutting test was stopped. The same experiment has been repeated for 3 times to get more accurate result. 


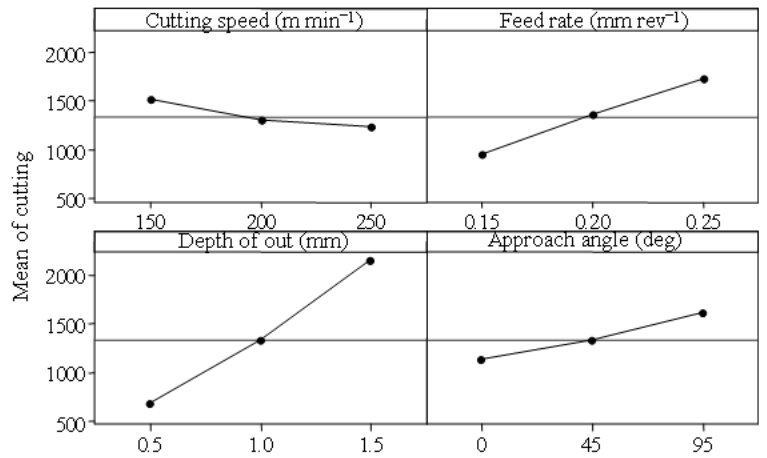

(a)

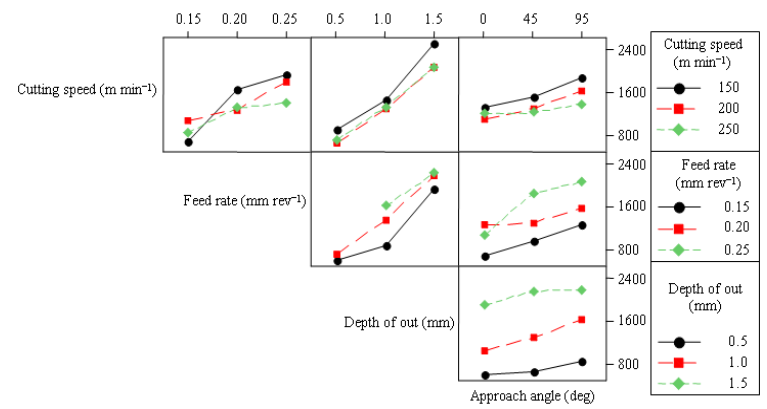

(b)

Fig. 3: Main effect (data means) for cutting force, (a): Linear plot (data means) for cutting force $(\mathrm{N})$ and (b): Interaction plot (data means) for cutting force $(\mathrm{N})$

Regression module: The cutting force (Linear + interaction) model is:

$$
\begin{aligned}
\mathrm{F}= & -7319+30.4 \mathrm{~V}+32581 \mathrm{f}+2199 \mathrm{~d}+5.0 \mathrm{~K}-138 \mathrm{Vf} \\
& -2.55 \mathrm{Vd}-0.0420 \mathrm{VK}-1417 \mathrm{fd}+40.8 \mathrm{fK}+0.22 \mathrm{dK}
\end{aligned}
$$

Where:

$\mathrm{F}=$ Cutting force $(\mathrm{N})$

$\mathrm{V}=$ Cutting speed $\left(\mathrm{m} \mathrm{min}^{-1}\right)$

$\mathrm{f}=$ Feed rate $\left(\mathrm{mm} \mathrm{rev}^{-1}\right)$

$\mathrm{d}=$ Depth of cut $(\mathrm{mm})$

$\mathrm{K}=$ Approach angle (deg)

From this linear equation, one can easily notice that the response y (cutting force) is affected significantly by the feed rate followed by depth of cut and then by feed rate with depth of cut and lastly, by the cutting speed and approach angle. Generally, the increase of feed rate, depths of cut and approach angle will cause the cutting force to become larger. On the other hand, the decrease in cutting speed will slightly cause increasing of cutting force as shown in Fig. 3.

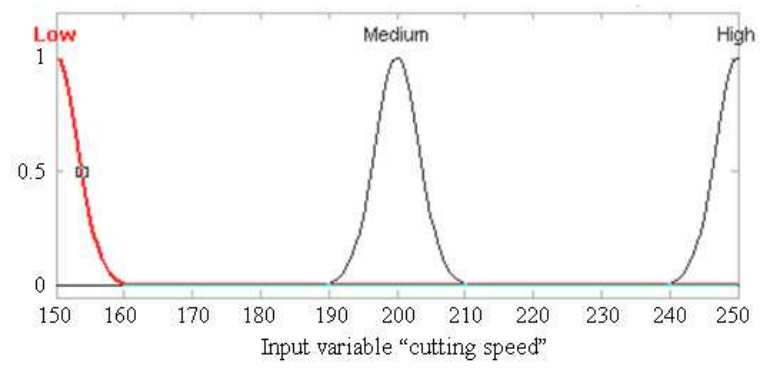

Fig. 4: Cutting speed membership function

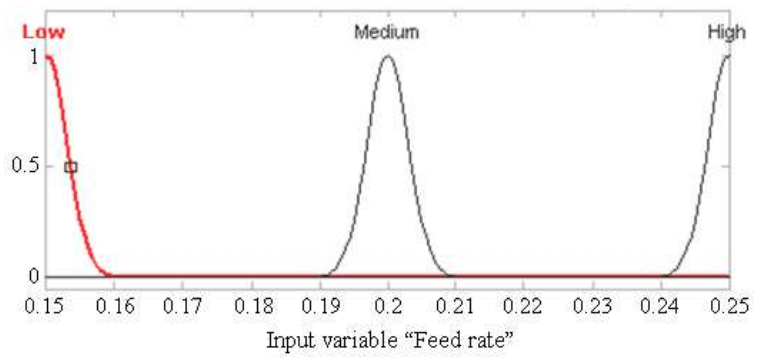

Fig. 5: Feed rate membership function

The proposed linear equation is valid only for cutting ceramic tools with a (-6) rake angle within the cutting conditions range used in the experimentation as shown in Table 4.

Fuzzy logics for the turning operation: The fuzzy model that has been designed for predicting cutting force for the turning operation uses four inputs and one output. Cutting speed, feed rate, depth of cut and approach angle are the inputs and cutting force is the output of the system. The first step in establishing the algorithm for selecting the cutting condition is to choose the shape of fuzzy membership functions or fuzzy sets for the process variables based upon experimental data. The system is based on the interrelationship that exists for machining nickel based Hastelloy C-276 material between its cutting speed (input 1) feed rate (input 2), depth of cut (input 3), approach angle (input 4) and the corresponding cutting force (output). Well distributed and bell-shape is used for the membership function for the input and the output variables. The membership functions for each fuzzy set for input fuzzy variables and for output fuzzy variable are shown in Fig. 4-8, respectively.

Rule-based fuzzy relations: Fuzzy rules are a set of linguistic statements which establishes the relationship between the input and the output in a fuzzy system. They are defined based on experimental work. 
Am. J. Engg. \& Applied Sci., 3 (1): 207-213, 2010

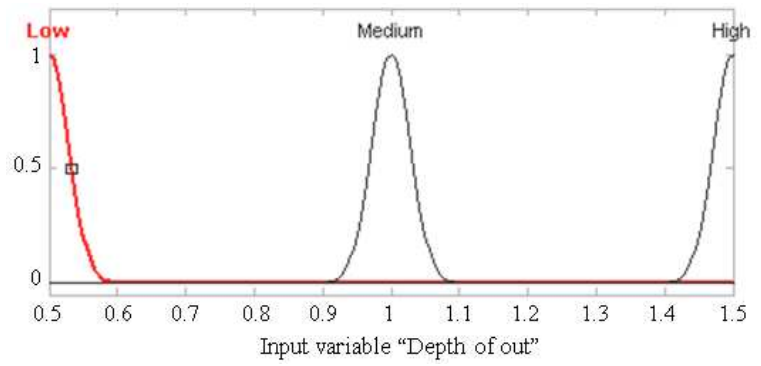

Fig. 6: Depth of cut membership function

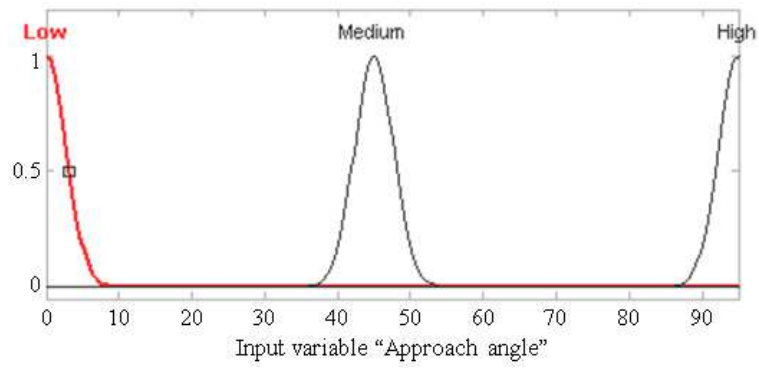

Fig. 7: Approach angle membership function

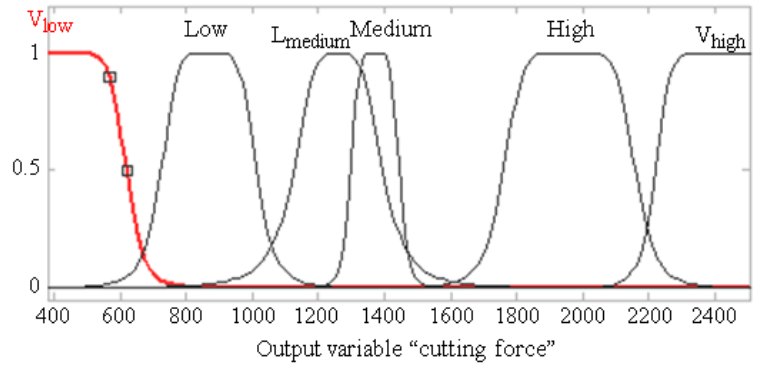

Fig. 8: Cutting force membership function

Table 6: Fuzzy rules in linguistic form

1. IF Cutting speed is (medium) and feed rate is (low) and depth of cut is (medium) and approach angle is (low) then Cutting force is (very low) (1)

2. IF Cutting speed is (high) and feed rate is (high) and depth of cut is (medium) and approach angle is (medium) then Cutting force is (medium) (1)

3. IF Cutting speed is (medium) and feed rate is (medium) and depth of cut is (low) and approach angle is (medium) then Cutting force is (very low) (1)

27. IF Cutting speed is (low) and feed rate is (high) and depth of cut is (medium) and approach angle is (medium) then Cutting force is (high) (1)

The number of fuzzy rules in a fuzzy system is related to the number of fuzzy sets for each input variable. In this study, there are four input variables which are classified into twenty seven fuzzy sets and there are twenty seven cutting force states to be determined. The "and" and "or" used in the rules will apply to the fuzzy "and" and "or" operations, respectively. Table 6 shows a few examples of fuzzy rules in a linguistic form.

\section{RESULTS AND DISCUSSION}

There are two methods commonly used to yield the aggregation of the rules. They are .Max-Min Inference Method and Max-Product Method. For the first method, all the fuzzy "and" and "or" operations are applied into all the input's value of the corresponding fuzzy sets (Hashmia et al., 2003). Applying a fuzzy "and" operation will yield a result that is the minimum of the fuzzy value of the number of input variables. The aggregation of the rule will be the truncation of the output fuzzy set. This method is applied to all rules to obtain the final result which gives the final shape of the output fuzzy membership function after aggregation of all the rules, respectively. Then the union operation is applied to all the output fuzzy sets to yield the final fuzzy set. The Max-Product method is similar to MaxMin Inference Method, the only difference being the aggregation of the rules, one would multiply the output fuzzy set with the yielded result. The input 1 universe "cutting speed" should be partitioned according to the minimum and maximum values allowed to control the system. On this basis the universe of the cutting speed has been split in the range of 150-250, with any value above this range assumed to be infinity and a zero value implying that the cutting speed is almost a minimum value. The value of 150 is assigned to "minimum cutting speed" and the value of 250 to "maximum cutting speed". In a similar manner the universe of the input 2 (feed rate), input 3 (depth of cut), input 4 (approach angle) and output (cutting force has been partitioned according to the range of predicted cutting force 300-2700. It is assumed that the value of 300 is assigned to "min. force" and the value of 2700 is assigned to "max. Force", for any output speed range. Table 7 shows the ranges of cutting force for min. and max values from response surface and fuzzy outputs.

Values plotted for experimental cutting force versos the predicted cutting force by response surface and fuzzy logics as shown in Fig. 7. The standard deviation is $\approx$ $10 \%$ for predicted response surface and $8.8 \%$ for fuzzy logics. Furthermore, the standard deviation error is $1.949 \%$ for response surface and $1.694 \%$ for predicted cutting force by fuzzy logics. The differences on some points can be explained by the other effects such as chatter, burr formation and tool wear. This is due to some high values of cutting force measured due experiments affected by these characterize (Khidhir and Mohamed, 2009) On the other hand the matching of nickel based Hastelloy C-276 can be controlled for all of most values using fuzzy logics and can be acceptable more than response surface. 
Am. J. Engg. \& Applied Sci., 3 (1): 207-213, 2010

Table 7: Fuzzy output for Cutting speed values from 27 rules

\begin{tabular}{lrrr}
\hline Sq. & $\begin{array}{c}\text { Experiment } \\
\text { cutting } \\
\text { force }(\mathrm{N})\end{array}$ & $\begin{array}{l}\text { Predicted cutting } \\
\text { force by response } \\
\text { surface }(\mathrm{N})\end{array}$ & $\begin{array}{c}\text { Predicted cutting } \\
\text { force by fuzzy } \\
\text { logics }(\mathrm{N})\end{array}$ \\
\hline 1 & 668.505 & 985,92 & 501.3 \\
2 & 1381.373 & 1221,32 & 1372.0 \\
3 & 606.011 & 461,32 & 501.3 \\
4 & 380.490 & 370,095 & 501.3 \\
5 & 1364.296 & 1429,32 & 1372.0 \\
6 & 544.242 & 657,27 & 501.3 \\
7 & 864.196 & 913,52 & 868.3 \\
8 & 1076.446 & 1342,32 & 868.3 \\
9 & 2505.799 & 2226,87 & 2363.0 \\
10 & 1861.001 & 1803,32 & 1960.0 \\
11 & 1269.983 & 1244,32 & 1260.0 \\
12 & 630.641 & 675,52 & 501.3 \\
13 & 2063.732 & 1935,37 & 1960.0 \\
14 & 2218.662 & 2315,695 & 2363.0 \\
15 & 2059.986 & 1988,32 & 1960.0 \\
16 & 819.681 & 696,845 & 868.3 \\
17 & 1188.372 & 1176,62 & 1260.0 \\
18 & 954.248 & 845,32 & 501.3 \\
19 & 1919.988 & 1846,545 & 1960.0 \\
20 & 1260.270 & 1378,32 & 1260.0 \\
21 & 1918.558 & 1866,92 & 1960.0 \\
22 & 2206.936 & 2319,12 & 2363.0 \\
23 & 872.062 & 693,77 & 868.3 \\
24 & 1260.270 & 1378,32 & 1260.0 \\
25 & 1298.258 & 1151,62 & 1260.0 \\
26 & 1260.270 & 1378,32 & 1260.0 \\
27 & 1919.620 & 2075,32 & 1960.0 \\
\hline Average & & 11.26803 & 7.551248 \\
Stdv. & & 10.12889 & 8.805333 \\
Stdv. error & & 1.949306 & 1.694587 \\
\hline & & &
\end{tabular}

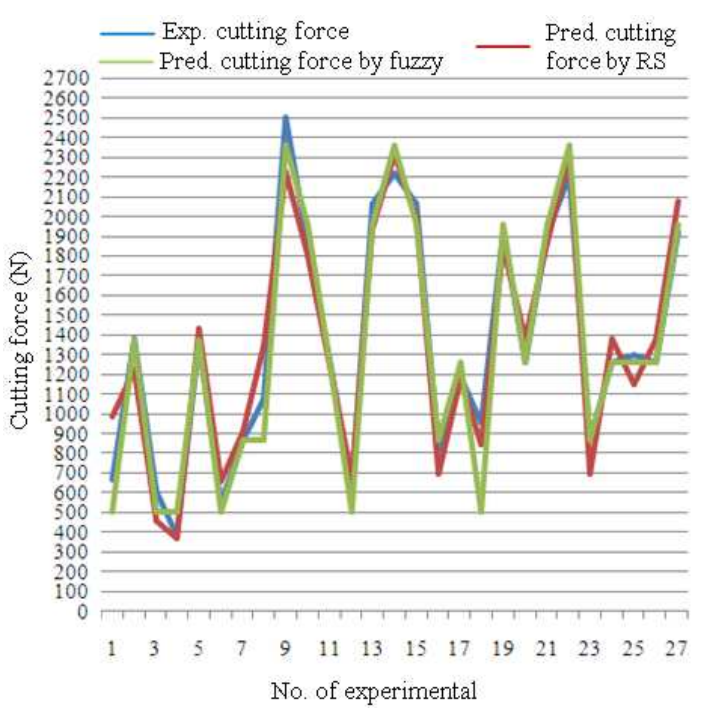

Fig. 9: Experimental cutting speed Vs predicted cutting speed by response surface and fuzzy logics

\section{CONCLUSION}

Fuzzy logic and response surface methodology are the latest optimization techniques that are being applied successfully in industrial applications for optimal selection of process variables in the area of machining. A review of literatures on optimization techniques has revealed that there are, in particular, successful industrial applications of design of experiment-based approaches for optimal settings of process variables. Response surface methodology are strong design techniques widely used in industries for making the product, process, insensitive to any uncontrollable factors such as Machining parameters. The application of a fuzzy logic based model for selecting cutting speed in a turning operation for predicting cutting force ranges is possible for the widest range of cutting parameters data produces the best match between predicted and experimental data. Reducing quality loss by designing the products and processes to be insensitive to variation in variables is a novel concept to manufacturers and quality engineers.

\section{REFERENCES}

Box, G.E.P. and K.P. Wilson, 1951. On the experimental attainment of optimum condition. J. Roy. Stat. Soc., 13: 1-45. http://www.garfield.library.upenn.edu/classics1981 /A1981MK45300001.pdf

Box, G.E.P. and P.V. Youle, 1955. The exploration and exploitation of response surfaces: An example of the link between the fitted surface and the basic mechanism of the system. Biometrics, 11: 287-323. http://www.jstor.org/pss/3001769

Box, G.E.P. and W.G. Hunter, 1957. Multifactor experimental designs for exploring response surfaces. Annal. Math. Stat., 28: 195-241. http://projecteuclid.org/DPubS ? service=UI\&versio $\mathrm{n}=1.0 \&$ verb$=$ Display\&handle $=$ euclid.aoms $/ 117770$ 7047

El Baradie, M.A., 1993. Surface roughness model for turning grey cast iron (154BHN). Proc. Inst. Mech. Eng. Part B. J. Eng. Manufact., 207: 43-54. http://cat.inist.fr/?aModele $=$ afficheN\&cpsidt $=4917886$

Hill, W.J. and W.G. Hunter, 1966. A review of response surface methodology: A literature survey. Technometrics, $\quad$ 8: 571-590. http://www.jstor.org/pss/1266632

Hashmia, K., I.D. Grahama, B. Mills and M.S.J. Hashmi, 2003. Adjustment approach for fuzzy logic model based selection of non-overlapping machining data in the turning operation. J. Mater. Process. Technol., 142: 152-162.

Hasegawa, M., A. Seireg and R.A. Lindberg, 1976. Surface roughness model for turning. Tribol. Int., 9: 285-289. 
Khidhir, B.A. and B. Mohamed, 2009. Selecting of cutting parameters from prediction model of cutting force for turning nickel based hastelloy C276 using response surface methodology. Eur. J. Sci. $\quad$ Res., $\quad 33$ : 526-536. http://www.eurojournals.com/ejsr_33_3_14.pdf

Mead, R. and D.J. Pike, 1975. A review of response surface methodology from a biometric viewpoint. Biometrics, 31: 803-851. http://www.ncbi.nlm.nih.gov/pubmed/1106790
Montgomery, D.C., 2001. Design and Analysis of Experiments. 5th Edition, John Wiley, New York, ISBN: 0-471-31649-0, pp: 684.

Sundaram, R.M. and B.K. Lambert, 1981. Mathematical models to predict surface finish in fine turning of steel, Part I. Int. J. Prod. Res., 19: 547-556.

http://www.informaworld.com/smpp/ftinterface?co ntent $=\mathrm{a} 778218342 \& \mathrm{rt}=0 \&$ format $=\mathrm{pdf}$ 